\title{
Autonomous Feature Tracing and Adaptive Sampling in Real-World Underwater Environments
}

\author{
Anwar Quraishi*, Alexander Bahr*, Felix Schill ${ }^{* *}$, Alcherio Martinoli*
}

\begin{abstract}
Applications of robots for gathering data in underwater environments has been limited due to the challenges posed by the medium. We have developed a miniature, agile, easy to carry and deploy Autonomous Underwater Vehicle (AUV) equipped with a suite of sensors for underwater environmental sensing. We have also developed a compact high resolution fast temperature sensing module for the AUV for microstructure and turbulence measurements in water bodies. In this paper, we describe a number of algorithms and subsystems of the AUV that enable autonomous real-world operation, and present the data gathered in an experimental campaign in collaboration with limnologists. We demonstrate adaptive sampling missions where the AUV could autonomously locate a zone of interest and adapt its trajectory to stay in it. Further, it could execute specific behaviors to accommodate special sensing requirements necessary to enhance the quality of the data collected. In these missions, the AUV could autonomously trace a feature and capture horizontal variation in various quantities, including turbidity and temperature fluctuations, allowing limnologists to study lake phenomena in an additional dimension.
\end{abstract}

\section{INTRODUCTION}

The opacity of water to electromagnetic waves limits remote sensing for measuring physical or biological parameters below the surface of water bodies. The underwater domain has therefore many applications for autonomous robots carrying in-situ sensing payloads to capture spatiotemporal phenomena. Generally, in terms of spatial coverage and resolution, the use of robots scales much better than static sensor nodes, since robots can potentially navigate to any desired measurement location [1]. Further, robots can specifically target regions of higher interest for gathering data [2], [3], [1]. Particularly for gathering data in water bodies, robots can have significant practical advantages. Currently, such data is obtained manually by deploying sensing probes or by installing static sensors, which requires a significant amount of effort and provides data at limited resolution. On the contrary, underwater robots can quickly obtain a large amount of data at unprecedented spatial resolutions. This allows capturing and studying various phenomena that take place in water bodies at smaller time and length scales.

In comparison to ground and aerial robots, examples of the use of underwater robots for sensing applications is limited, particularly in lakes. The reasons include harshness of underwater environments and high cost of commercially

\footnotetext{
* Distributed Intelligent Systems and Algorithms Laboratory (DISAL), School of Architecture, Civil and Environmental Engineering, École Polytechnique Fédérale de Lausanne (EPFL), 1015 Lausanne, Switzerland.

** Hydromea S.A., Lausanne, Switzerland.

This work was funded by the Swiss National Science Foundation under grant CRSII2_160726/1. http://disal.epfl.ch/research/AUVDistributedSensing.
}

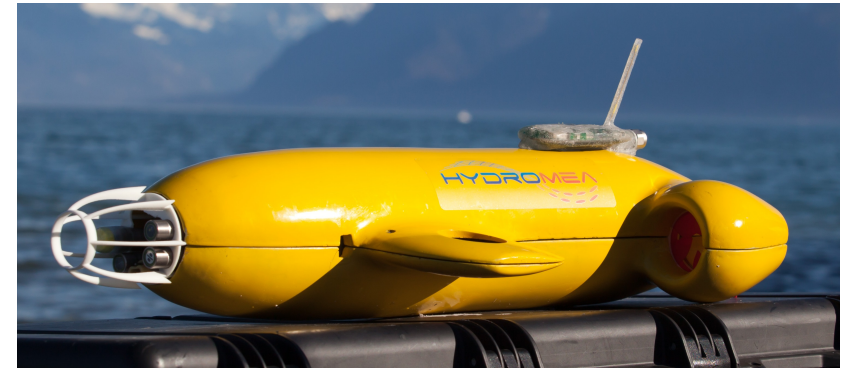

Fig. 1: The Vertex AUV with its sensor suite in the front, protected by a white cage. The AUV is about $70 \mathrm{~cm}$ long, weighs $7 \mathrm{~kg}$, and can be carried and deployed easily by one person.

available underwater robot platforms. Many of them are large in size and weight, which makes them difficult to deploy in lakes where large ships with cranes are generally not available. Some small-sized (roughly $0.5-1 \mathrm{~m}$ ) research prototypes do exist [4], [5], [6], but they typically have a very limited rated depth, and many of them have not yet been deployed in outdoor environments. One of the few commercially available small size robots are the Riptide UUV (Unmanned Undersea Vehicle) range of robots [7].

For our work, we used the Vertex AUV, a platform developed in our laboratory and currently manufactured and commercialized by Hydromea S.A ${ }^{1}$ The Vertex AUV is shown in Fig. 1 and more details about it can be found in [8]. It is small enough to be easily deployed and retrieved by one person, and was designed for distributed, cooperative multiAUV sensing in lakes and coastal areas. It has an integrated modular environmental sensor payload comprising a host of sensing probes, including a $400 \mathrm{~Hz}$ high resolution $(20 \mu \mathrm{K})$ temperature sensor module that was developed in-house. The high sampling frequency and resolution of the fast temperature sensor allows characterization of small-scale turbulence induced by underwater microbial activity, which was of particular interest for the research described in this paper. We use the AUV to capture lateral variation in temperature, turbidity, chlorophyll and various other quantities, which will help in advancing the understanding of physical and biological phenomena taking place in water bodies. In this paper, we collected measurements in a Swiss mountain lake within a thin bacterial layer, using an adaptive strategy.

Several approaches to various underwater sampling problems exist, and a number of them have been demonstrated experimentally in real environments. Zhang et al. [9] employed an AUV to detect a coastal upwelling front separating

${ }^{1}$ http://www.hydromea.com 
a stratified and an upwelling water column, and programmed the AUV to travel between the two regions in order to track the horizontal position of the front in one dimension over time. They use differences in temperature at two fixed depths during 'yo-yo' AUV trajectories as a feature to distinguish regions on either side of the boundary. [10] proposes a method for 3D front tracking, and demonstrates it with simulations. In [11], a drifter is used to track the location of an interesting feature such as an algal patch in flowing water. An AUV then executes a pre-planned survey pattern relative to the drifter position. An isotherm tracking method is demonstrated in [12], where the AUV performs a saw-tooth trajectory within a temperature envelope instead of a depth envelope. In [13], the AUV adjusts its buoyancy to control its depth and stay within a temperature bracket and track an isotherm. It does not use its propellers for motion during this time but drifts with the flow of water.

Our goal in this work is to track the boundary of a feature in a lake, and adaptively adjust the trajectory of the AUV to trace the feature and capture spatial data within it. The AUV can complement traditional limnological sensing methods by transporting the on-board sensing probes along a horizontal trajectory and capturing lateral variations at a high resolution. In particular, we want to take high-frequency, high-resolution temperature measurements within a thin bacterial layer, which will allow limnologists to determine the turbulence and mixing caused by the swimming motions of the bacteria that form the layer. The challenges in doing this are two-fold. Firstly, vibrations induced by the propellers can degrade the quality of the fast temperature sensor measurements. Secondly, the stratified bacterial layer is not uniformly spread throughout the lake but has varying depth and thickness.

We explain the experimental scenario and the practical challenges in detail in Section III. In Section III, we introduce the navigation, control and other subsystems implemented on the Vertex AUV that enable deployment in outdoor environments for limnological data-gathering missions. In the following Section (IV), we present our approach to face the aforementioned challenges and show the results gathered in the lake. Finally, we highlight the advantages the AUV brings to the field of limnology, by obtaining data at a high spatial resolution and allowing scientists to capture smallscale, fast changing phenomena.

\section{PROBLEM DEFINITION}

As a part of an interdisciplinary research project, we collaborate with limnologists and microbiologists to map physical, chemical and biological quantities with AUVs in lakes at high spatial resolutions (of the order of $1 \mathrm{~cm}$ vertically and $10 \mathrm{~cm}$ horizontally). The objective is to understand the interplay between small-scale physical processes and biological activity in water bodies. For this work, we gathered data from Lake Cadagno in Switzerland, which has a distinct biological phenomenon. It is a meromictic lake with a stratified thermocline layer at a depth of about $15 \mathrm{~m}$, which contains diverse species of anaerobic sulfurreducing photosynthetic bacteria [14], [15], as shown in Fig.

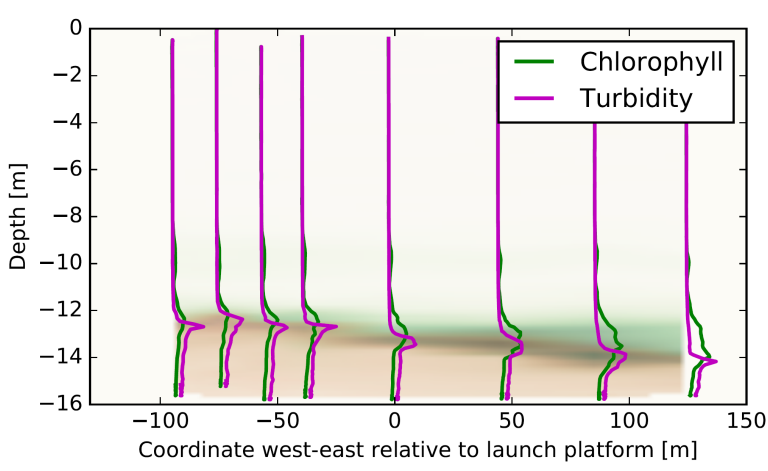

Fig. 2: A diagram of eight vertical profiles taken from an autonomous surface vehicle along an east-west transect during an afternoon in September 2016, showing the stratified bacterial layer (dark brown) below a layer rich in chlorophyll (green) in Lake Cadagno. The layer has varying thickness and depth and is characterized by a well-mixed thermocline and high turbidity.

2 The layer has a thickness of 0.25-2 $\mathrm{m}$. These bacteria can accumulate and increase the density of the local volume of fluid, which sinks and mixes with the surrounding water, while upward swimming bacteria help maintain water motion. This phenomenon, called bio-convective mixing, generates smallscale turbulent eddies within the stratified bacterial layer [16]. These eddies can be characterized by observing the spatial spectra of temperature measurements. It is important to understand the activity of these sulfur bacteria and mixing processes in order to study their effect on the lake ecosystem.

Currently, limnological measurements in lakes are performed with the help of vertical profilers [17] deployed from various discrete locations, and moored static sensors [18]. Vertical profilers are devices containing a suite of sensors that sink vertically at a controlled speed. In our experiments, the sensors would cross through the bacterial layer and measure within it while sinking. As mentioned earlier, these devices can obtain data at a high vertical resolution, but the horizontal resolution is limited since they cannot traverse the water body laterally.

The AUV can greatly complement these traditional methods by gathering data at high lateral resolutions. The length scales of the turbulent eddies within the bacterial layer are similar to the thickness of the layer, i.e., they can be as small as $0.25 \mathrm{~m}$. The fast temperature sensor on the AUV measures at $400 \mathrm{~Hz}$, and the AUV cruise speed is limited to $1 \mathrm{~m} / \mathrm{s}$, which implies that the temperature measurements are taken every $2.5 \mathrm{~mm}$, which is adequate for capturing the turbulent eddies. However, there are a number of practical challenges in sampling with an AUV. Firstly, the temperature fluctuations due to the small-scale turbulence within the bacterial layer are extremely weak, in the submillikelvin range. The fast temperature sensor on the AUV (see Section III-D) is capable of resolving those fluctuations. However, vibrations induced by the AUV propellers can also cause interference in the temperature measurements due to the extremely high sensitivity of the sensor, which can potentially corrupt the turbulence measurements. To mitigate this effect, we implemented a behavior that turns off all propellers for a short duration at a suitable time, and 


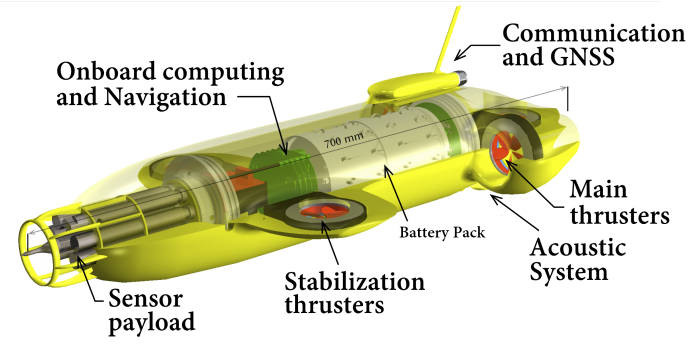

Fig. 3: Various subsystems and thrusters of the AUV. The fifth thruster is mounted vertically in the tail of the AUV.

let the AUV drift forward due to its inertia and capture spatial temperature measurements without motor vibrations. Secondly, the thickness and depth of the bacterial layer varies over the lake and with time of the day (see Fig. 2). Data from traditional sensing methods can provide localized information about the layer depth, but this was found to be inadequate to guarantee that preplanned, horizontal AUV trajectories would always trace the layer. Therefore, we process sensor measurements in real-time on-board the AUV to adaptively adjust the trajectory and trace the layer.

\section{RELEVAnt AUV SUbSYSTEMS}

\section{A. Actuation}

Five thrusters provide propulsion and attitude control on the Vertex AUV, as shown in Fig. 3. Two lateral horizontal thrusters provide propulsion and yaw control. Two lateral vertical thrusters in the winglets in the front provide roll control. They also provide pitch control along with the third vertical thruster mounted in the rear of the AUV.

\section{B. Navigation}

An Extended Kalman Filter (EKF) framework is used for navigation, which fuses inertial and magnetic compass measurements, and a comprehensive model of the dynamics of the AUV. The dynamics model takes into account the rotational speed of the propellers, inertia of the AUV, as well as the effect of buoyancy and viscous drag on the body of the AUV. The EKF is complemented by a Global Navigation Satellite System (GNSS) position fix whenever the AUV is on the surface. For all the data gathering missions, the AUV is effectively performing dead reckoning, aided by the compass for its heading, Inertial Measurement Unit (IMU) for attitude, dynamic model for velocity, and a depth sensor. An acoustic positioning system for underwater localization is under development for future missions and was not used during this work. This results in position errors accumulating over the length of the trajectory. Although this does imply that the spatial data gathered is associated with less accurate absolute positions, for a number of purposes the effect on the quality of the dataset is not significant. This is because the focus is on relative positions between spatial sampling points and spatial variations of various quantities rather than accurate absolute positions themselves.

\section{Active depth control}

The AUV is slightly more buoyant than its weight, which implies that there is a constant net force that tends to displace

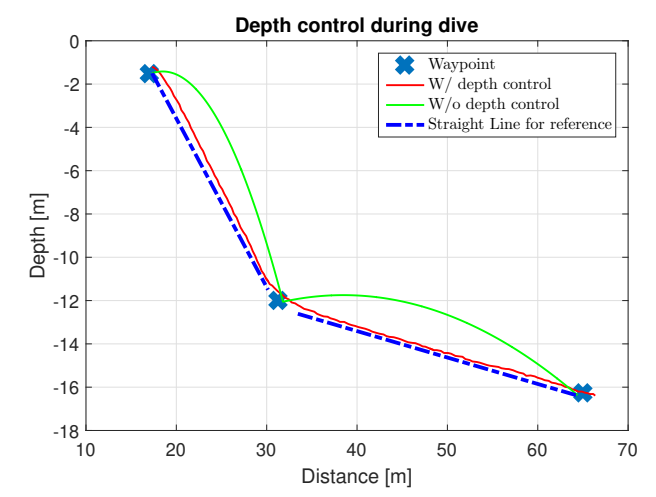

Fig. 4: AUV trajectory with and without depth control. Without active depth control, the AUV can reach the programmed waypoints, but the trajectory will not be a straight line. This plot is from a simulation.

the body in the upward direction. Therefore, in order to follow a planned trajectory or to hold a fixed position, the depth must be actively regulated (see Fig. (4). The required depth, $d_{\mathrm{cmd}}$ is computed by the navigation module. For hovering at a certain position, $d_{\mathrm{cmd}}$ is the depth of that position. When navigating between two waypoints, from $\vec{W}_{1}$ to $\vec{W}_{2}, d_{\text {cmd }}$ is computed in a way that the trajectory between the waypoints is a straight line. That is, $d_{\text {cmd }}$ varies linearly between $W_{1 z}$ and $W_{2 z}$ with the distance covered by the AUV in the horizontal plane. If the current position of the AUV is $\vec{x}$,

$$
d_{\mathrm{cmd}}=W_{1 z}+\left(W_{2 z}-W_{1 z}\right) \frac{\mathrm{D}_{x y}\left(\vec{x}, \vec{W}_{1}\right)}{\mathrm{D}_{x y}\left(\vec{W}_{2}, \vec{W}_{1}\right)},
$$

where $\mathrm{D}_{x y}\left(\vec{v}_{1}, \vec{v}_{2}\right)$ is the distance between vectors $\vec{v}_{1}$ and $\vec{v}_{2}$ projected on the horizontal plane. Then, the depth error is

$$
d_{\text {err }}=d_{\mathrm{cmd}}-d_{\text {actual }} \text {. }
$$

When stationary or at low speeds, the vertical thrusters are used to regulate depth. This consumes a relatively large amount of energy. When moving forwards with a speed higher than a threshold, it is more efficient to adjust the pitch angle downward to regulate depth, using the AUV hull and the winglets to generate a vertical force. We have a smooth transition between the two controllers around the threshold speed $s_{0}$, which was set to $0.5 \mathrm{~m} / \mathrm{s}$, half of the maximum allowed cruise speed. The inputs to the PID controller for pitch, $\phi_{\mathrm{cmd}}$ and heave command to the vertical thrusters, $H_{\mathrm{cmd}}$ are computed as

$$
\begin{aligned}
\phi_{\mathrm{cmd}} & =f_{m i x} \cdot K_{\phi} \cdot d_{e r r} \\
H_{\mathrm{cmd}} & =\left(1-f_{m i x}\right) \cdot K_{H} \cdot d_{e r r}
\end{aligned}
$$

where $K_{\phi}$ ans $K_{H}$ are constant parameters and the mixing factor $f_{\text {mix }}$ is computed as a function of the AUV velocity $\vec{v}$ and threshold speed $s_{O}$ as

$$
f_{\text {mix }}=\frac{1}{2}+\frac{1}{2} \cdot \frac{K \cdot\left(\|\vec{v}\|-s_{o}\right)}{\sqrt{\left(1+K^{2}\left(\|\vec{v}\|-s_{o}\right)^{2}\right)}} .
$$

$f_{m i x} \in[0,1]$ and $f_{m i x}=0.5$ when the AUV speed $\|\vec{v}\|$ is equal to $s_{o}$. The parameter $K$ controls the smoothness of the controller transition. 


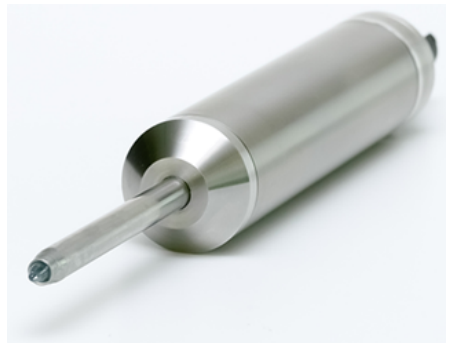

Fig. 5: The fast temperature sensing module. It is $20 \mathrm{~cm}$ long and $2.5 \mathrm{~cm}$ in diameter.

\section{Sensing}

The AUV is equipped with a fast temperature sensor module and a YSI EXO2 water quality measurement sonde [19]. The YSI sonde is a commercially available modular system which can be configured with up to seven different sensing probes. The fast temperature sensor module has been developed in-house and can measure at a sampling rate of up to $400 \mathrm{~Hz}$. It is enclosed in a package compact enough to be integrated into the AUV (see Fig. 55). While not calibrated to be accurate, this sensor has a resolution of $20 \mu \mathrm{K}$, which makes it suitable for capturing microstructure- and turbulenceinduced temperature fluctuations. The YSI sonde has an additional temperature sensor for reference that has lower resolution and sampling rate, but an accuracy of $10 \mathrm{mK}$. The fast temperature module also includes a 9-axis inertial measurement unit for measuring the orientation and vibrations of the temperature sensor. This module was compared to existing fast temperature sensors from Rockland Scientific with measurements in a flow channel and in an outdoor environment, in collaboration with limnology researchers, and was found to have similar performance.

During this work, the AUV was configured with turbidity, blue-green algae+chlorophyll, CTD (conductivity, temperature, depth), and dissolved oxygen sensors, along with the fast temperature module. All sensor data is sent to the AUV control and navigation modules in real-time, and logged along with AUV data. This allows us to process the data on-board and use it for real-time control. Further, this also allows us to post-process sensor data in conjunction with AUV operational data in order to evaluate and implement adaptive behaviors.

\section{E. Mission Planner}

The mission planner software consists of an interface running on a computer that communicates with the AUV over radio. It allows the user to upload mission plans that comprise of a list of waypoints, specified in terms of latitude, longitude, depth, and other parameters. The mission planner uses a bathymetric map of the lake to limit depth parameters of all waypoints accordingly. There is no communication with the AUV when it is submerged.

\section{Methodology And Results}

A number of AUV missions were performed over a period of three days in Lake Cadagno. Data were gathered along several trajectories, which were planned in collaboration with limnologists. Fig. 6 shows a plot of the mission trajectories.

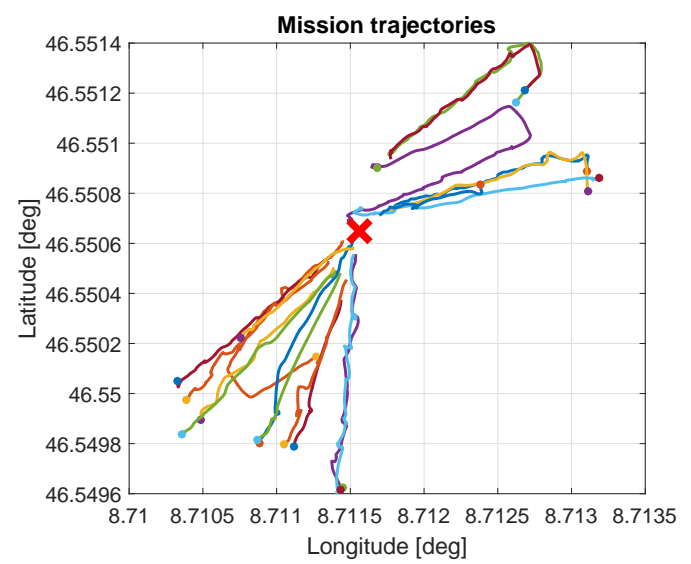

Fig. 6: AUV trajectories at Lake Cadagno from 19 separate missions, projected on the horizontal plane. The red cross marks the launch point of the missions.

The horizontal distance to be covered in each of those missions was about $100 \mathrm{~m}$, and the maximum cruise speed of the AUV was set to $1 \mathrm{~m} / \mathrm{s}$. The initial missions were fully pre-planned, and the subsequent ones had an adaptive component in order to autonomously detect and trace the bacterial layer. All missions were completely autonomous; given that there was no communication with the AUV while it was underwater, manually controlled missions were not possible.

\section{A. Preliminary autonomous missions}

For preliminary missions, we planned several 'yo-yo' dive trajectories between a specified depth envelope as shown in Fig. 8 . The depth parameters were chosen to be conservative enough to capture the bacterial layer during the dives. The AUV then executed the trajectories autonomously. The sensor data from one of these missions is shown in Fig. 8a. The twin peaks in turbidity and chlorophyll during each yoyo dive segment show that the AUV crossed through the bacterial layer in downward and upward directions of the dive. However, in these missions, the lateral coverage within layer is limited. We performed several such trajectories to capture spatial data over an area of the lake.

We then planned a shallow dive mission shown in Fig. $8 \mathrm{~b}$ where the AUV descends vertically over a long horizontal distance. This was done in an attempt to traverse through the layer over a longer horizontal distance. Yet, given that the layer thickness and depth is not constant, in many of the missions, only a short segment of the trajectory actually passed through the layer.

Fig. 8c shows a plot of chlorophyll concentration and turbidity against depth over one of the yo-yo trajectories. We see that there is a sharp increase in both these quantities within the layer, at a depth of around $16 \mathrm{~m}$. This observation is consistent with the results reported in [14] stating that the bacterial layer is characterized by higher concentrations of chlorophyll and a turbidity maximum.

\section{B. Adaptive sampling}

Given the sharp variation in turbidity shown in the previous section, a simple threshold on turbidity measurements was 


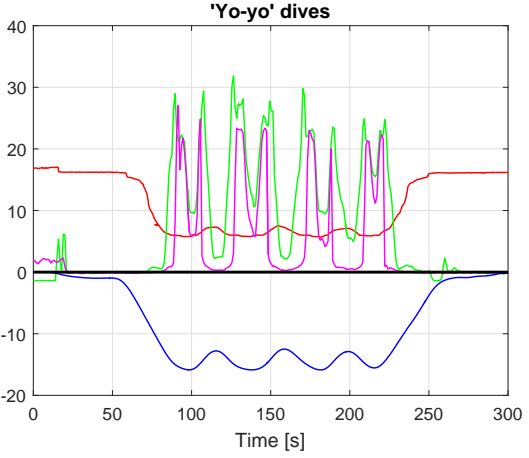

(a)

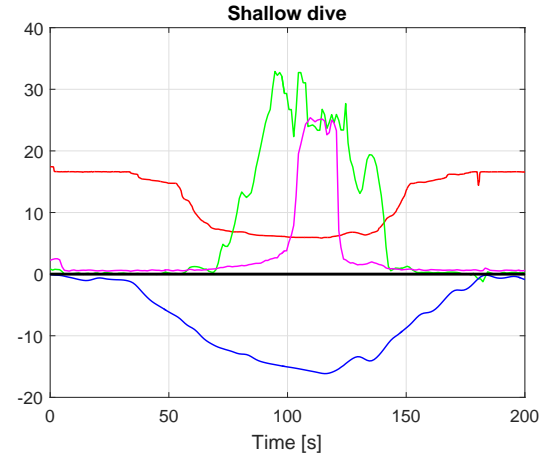

(b)

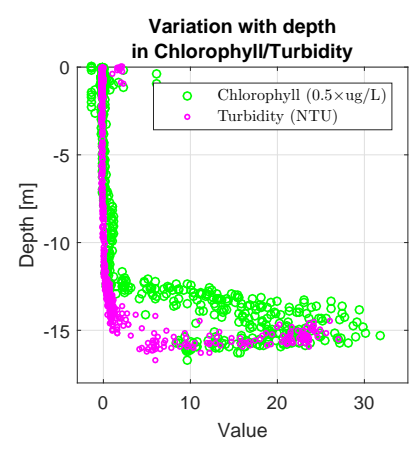

(c)

Fig. 8: Pre-programmed dive missions to measure through the bacterial layer. Plots (a) and (b) show the depth of the AUV over time and the corresponding sensor measurements. (a) shows a yo-yo dive mission between a depth envelope of $12 \mathrm{~m}$ and $16 \mathrm{~m}$. The twin peaks in chlorophyll and turbidity show the crossing of the AUV through the bacteria layer. (b) shows a shallow dive from $14 \mathrm{~m}$ to $16 \mathrm{~m}$ over a horizontal distance of $15 \mathrm{~m}$. (c) shows variation in chlorophyll / turbidity with depth. There is a sharp rise in these quantities within the bacterial layer.

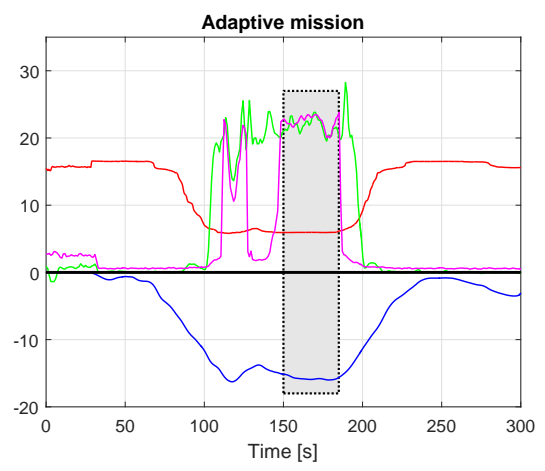

Fig. 9: Sensor data from an adaptive mission. The shaded region marks the segment where trajectory is adaptively adjusted. A high value of turbidity shows that the robot traversed within the layer.

used to detect the bacterial layer. During the adaptive missions, the first two waypoints were specified with depth parameters conservative enough to encompass the layer, which allowed the AUV to detect the local layer boundaries. We then used the newly implemented type of waypoints where the horizontal positions are pre-specified by the user but the depth is set automatically during the mission based on turbidity measurements. The turbidity was monitored continuously to update the layer boundary estimates in case the AUV left the layer. Then, the AUV would adjust its trajectory in real-time and attempt to stay within the layer for the rest of the mission. The high value of turbidity during the mission, as is seen in Fig. 9. indicates that the AUV traced the bacterial layer.

\section{Burst mode}

Tracing the layer with adaptive sampling is sufficient for gathering spatial variation in all quantities except fast temperature. Since temperature fluctuations due to the turbulence are very weak, the temperature measurements can be easily contaminated due to the vibrations induced by the AUV propellers. To mitigate this effect, we implemented another new type of waypoint, which we call burst waypoint. The AUV aimed for this waypoint at the maximum possible cruise

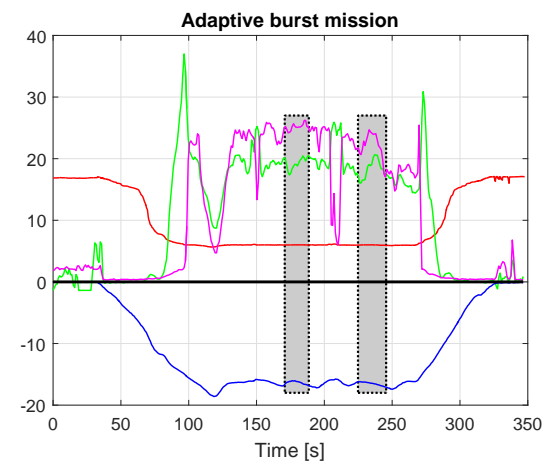

Fig. 10: Sensor data from mission with burst mode waypoints. The shaded regions indicate the burst segments which lasted for $20 \mathrm{~s}$, during which the robot drifted forward within the layer due to inertia.
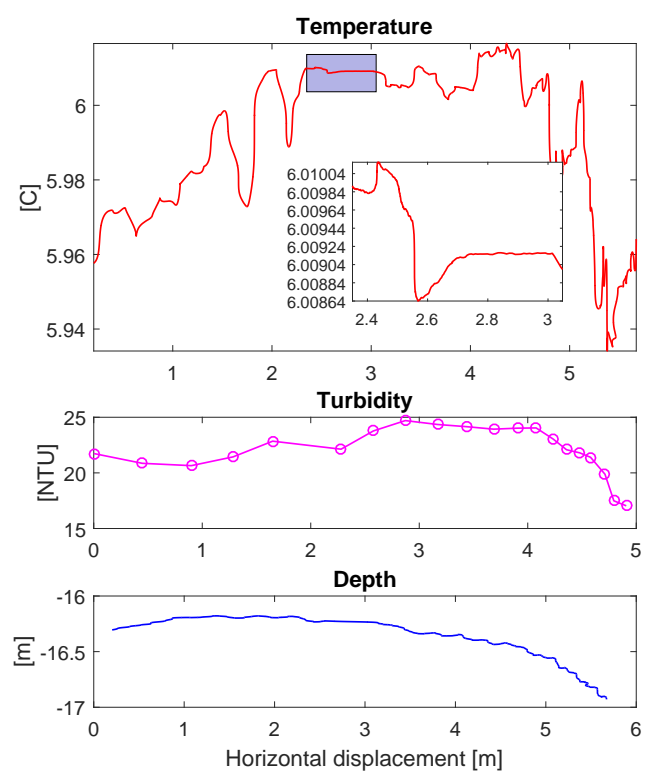

Fig. 11: Sensor data from a burst segment. A zoomed-in view of the shaded section of the temperature plot is shown. 
speed, and on reaching it, it turned off all motors for a specified duration of time, usually $20 \mathrm{~s}$. It drifted forward due to inertia and continued to sample data without the motor vibrations. The next step was to combine the adaptive and burst features to sample data within the layer with motors off. This was achieved by setting the depth of the burst waypoint automatically by monitoring the turbidity measurements in real-time, as explained in the previous section. This allowed the AUV to drift horizontally within the layer with its motors off, thus capturing the lateral variation in temperature required for studying the small-scale turbulent eddies. In the data from an adaptive burst mission shown in Fig. 10, there are two burst segments, each with a duration of $20 \mathrm{~s}$. An expanded view of one of the burst segments is shown in Fig. 11. The horizontal displacement of the AUV (estimated by the on-board EKF) during this time is about $6 \mathrm{~m}$. A high value of turbidity indicates that the robot traced the bacterial layer. A zoomedin view of the temperature plot shows the minute fluctuations in temperature. The bio-induced eddies can be characterized by studying the spatial spectra of this temperature signal.

\section{CONCLUSION}

We implemented autonomous feature tracing and adaptive sampling behaviors for gathering data in real-world environments, and demonstrated the capability of the Vertex AUV to execute them. We collaborated with limnologists to gather data from Lake Cadagno in Switzerland. The AUV could successfully trace the compact bacterial layer in the lake to sample various quantities at a high lateral resolution. Further, in separate missions, the AUV could turn off the motors during the mission within the layer and drift forward due to inertia. This was done to avoid motor-induced vibrations from corrupting the fast temperature sensor measurements, thereby enhancing the quality of temperature data. High lateral resolution data gathered within the bacterial layer would complement measurements taken using standard limnological methods. This would allow studying various biological and physical processes in an additional dimension. In summary, our contributions were (i) implementing active depth control on the AUV to counter the buoyant force and hold depth or maintain a planned trajectory, (ii) processing sensor data on the AUV in real time to locate and stay within a zone of interest, and (iii) implementing and executing specific behaviors that enhance the quality of the data gathered.

During this work, the AUV had no external position reference (except for the depth sensor) for underwater localization, and essentially performed inertial navigation. We are currently developing an acoustic localization system, which will provide underwater positioning in an absolute frame for future field experiments.

\section{ACKNOWLEDGMENTS}

The authors are thankful to Prof. Alfred J. Wüest, Dr. Damien Bouffard and Oscar Sepúlveda of the Physics of Aquatic Systems Laboratory at EPFL for their collaboration during the field experiments at Lake Cadagno and for their help in evaluating the performance of the AUV's fast temperature sensor.

\section{REFERENCES}

[1] R. Wang, M. Veloso, and S. Seshan, "Active sensing data collection with autonomous mobile robots," in IEEE International Conference on Robotics and Automation, 2016, pp. 2583-2588.

[2] D. E. Soltero, M. Schwager, and D. Rus, "Decentralized path planning for coverage tasks using gradient descent adaptive control," The International Journal of Robotics Research, vol. 33, no. 3, pp. 401-425, 2014.

[3] G. A. Hollinger and G. S. Sukhatme, "Sampling-based robotic information gathering algorithms," The International Journal of Robotics Research, vol. 33, no. 9, pp. 1271-1287, 2014.

[4] C. Osterloh, T. Pionteck, and E. Maehle, "MONSUN II: A small and inexpensive AUV for underwater swarms," in 7th German Conference on Robotics, 2012, pp. 325-330.

[5] A. Amory and E. Maehle, "SEMBIO - a small energy-efficient swarm AUV," in MTS/IEEE OCEANS, 2016, DOI: 10.1109/OCEANS.2016.7761458 (7 pages).

[6] A. Hackbarth, E. Kreuzer, and E. Solowjow, "Hippocampus: A micro underwater vehicle for swarm applications," in IEEE/RSJ International Conference on Intelligent Robots and Systems, 2015, pp. 2258-2263.

[7] "Micro-UUV from Riptide," http://riptideas.com/micro-uuv/ accessed: 2017-02-15.

[8] F. S. Schill, A. Bahr, and A. Martinoli, "Vertex: A new distributed underwater robotic platform for environmental monitoring," in International Symposium on Distributed Autonomous Robotic Systems, 2016. Springer Proceedings in Advanced Robotics, 2018, To appear. [Online]. Available: https://infoscience.epfl.ch/record/225323

[9] Y. Zhang, M. A. Godin, J. G. Bellingham, and J. P. Ryan, "Using an autonomous underwater vehicle to track a coastal upwelling front," IEEE Journal of Oceanic Engineering, vol. 37, no. 3, pp. 338-347, 2012.

[10] S. Petillo, H. Schmidt, P. Lermusiaux, D. Yoerger, and A. Balasuriya, "Autonomous \& adaptive oceanographic front tracking on board autonomous underwater vehicles," in IEEE OCEANS, 2015, DOI: 10.1109/OCEANS-Genova.2015.7271616 (10 pages).

[11] J. Das, F. Py, T. Maughan, T. OReilly, M. Messie, J. Ryan, K. Rajan, and G. S. Sukhatme, "Simultaneous tracking and sampling of dynamic oceanographic features with autonomous underwater vehicles and lagrangian drifters," in International Symposium on Experimental Robotics. Springer Tracts in Advanced Robotics, vol. 79, 2014, pp. 541-555.

[12] F. Cazenave, Y. Zhang, E. McPhee-Shaw, J. G. Bellingham, and T. P. Stanton, "High-resolution surveys of internal tidal waves in monterey bay, california, using an autonomous underwater vehicle," Limnology and Oceanography: Methods, vol. 9, no. 12, pp. 571-581, 2011.

[13] Y. Zhang, B. Kieft, M. J. Stanway, R. S. McEwen, B. W. Hobson, J. G. Bellingham, J. P. Ryan, T. C. O’Reilly, B. Y. Raanan, M. Messi, J. M. Smith, and F. P. Chavez, "Isotherm tracking by an autonomous underwater vehicle in drift mode," IEEE Journal of Oceanic Engineering, vol. 42, no. 4, pp. 808-817, 2017.

[14] M. Tonolla, S. Peduzzi, A. Demarta, R. Peduzzi, and D. Hahn, "Phototropic sulfur and sulfate-reducing bacteria in the chemocline of meromictic Lake Cadagno, Switzerland," Journal of Limnology, vol. 63, no. 2, pp. 161-170, 2004.

[15] M. Tonolla, S. Peduzzi, D. Hahn, and R. Peduzzi, "Spatio-temporal distribution of phototrophic sulfur bacteria in the chemocline of meromictic Lake Cadagno (Switzerland)," FEMS Microbiology Ecology, vol. 43, no. 1, pp. 89-98, 2003.

[16] T. Sommer, F. Danza, J. Berg, A. Sengupta, G. Constantinescu, T. Tokyay, H. Bürgmann, Y. Dressler, O. Sepúlveda Steiner, C. Schubert et al., "Bacteria-induced mixing in natural waters," Geophysical Research Letters, vol. 44, no. 18, pp. 9424-9432, 2017.

[17] "VMP-500 Turbulence Profiler from Rockland Scientific," http:// rocklandscientific.com/products/profilers/vmp-500/ accessed: 2017-0906.

[18] "Nemo moored platform for turbulence measurements from rockland scientific," http://rocklandscientific.com/products/moored-systems/ nemo/ accessed: 2017-09-06.

[19] "YSI EXO2 Multiparameter Sonde," http://www.ysi.com/EXO2 accessed: 2017-09-06. 